\title{
Dynamic and Transparent Analysis of Commodity Production Systems
}

\author{
Aristide Fattori $^{\dagger} \quad$ Roberto Paleari ${ }^{\dagger}$ \\ \{aristide,roberto\}@security.dico.unimi.it \\ Lorenzo Martignoni ${ }^{\ddagger}$ \\ Mattia Monga \\ lorenzo.martignoni@uniud.it \\ mattia.monga@unimi.it
}

\author{
Dip. di Informatica e Comunicazione ${ }^{\dagger}$ \\ Università degli Studi di Milano \\ I-20135 Milan, Italy
}

\author{
Dip. di Fisica $\ddagger$ \\ Università degli Studi di Udine \\ I-33100 Udine, Italy
}

\begin{abstract}
We propose a framework that provides a programming interface to perform complex dynamic system-level analyses of deployed production systems. By leveraging hardware support for virtualization available nowadays on all commodity machines, our framework is completely transparent to the system under analysis and it guarantees isolation of the analysis tools running on its top. Thus, the internals of the kernel of the running system needs not to be modified and the whole platform runs unaware of the framework. Moreover, errors in the analysis tools do not affect the running system and the framework. This is accomplished by installing a minimalistic virtual machine monitor and migrating the system, as it runs, into a virtual machine. In order to demonstrate the potentials of our framework we developed an interactive kernel debugger, nicknamed HYPERDBG. HyperDbG can be used to debug any critical kernel component, and even to single step the execution of exception and interrupt handlers.
\end{abstract}

\section{Categories and Subject Descriptors}

D.2.5 [Software Engineering]: Testing and DebuggingDebugging aids, Monitors, Tracing; D.4.9 [Operating Systems]: Systems Programs and Utilities

\section{General Terms}

Verification

\section{Keywords}

hardware virtualization, debugging, system analysis

\section{INTRODUCTION}

Operating systems are peculiar and very complex pieces of software whose internals are critically vital for a system: a failure, or a bottleneck, in any of their parts can lead to

To appear in the $25^{\text {th }}$ IEEE/ACM International Conference on Automated Software Engineering, Antwerp, Belgium, 20-24 September 2010 catastrophic consequences. Therefore, special care is needed to develop, analyze, test, and profile them. To simplify their task, developers and analysts rely on a large variety of tools and analysis techniques. Some of them are specific for studying static properties of the operating system, while others are more specific for studying dynamic properties. In particular, the latter class of tools and techniques is nowadays very popular among kernel developers and analysts because it allows them to collect the information very quickly, while hiding many of the intricacies of the kernel, and can even be used on running production systems.

Existing approaches for dynamic analysis of operating systems (e.g., debugging, profiling, and tracing) can be roughly classified in two groups: kernel-based and VMM-based. The approach taken by the first group is to include some component into the kernel in order to intercept all the events of interest (e.g., the creation of a new process, the execution of a system call, and the execution of a kernel function) and to execute a specific action when such events occur $[2,11$, $14,18,20]$. This solution requires the installation of specific hooks in the kernel to monitor run-time events and it might be very difficult to apply to operating systems that do not natively offer facilities for dynamic analysis, especially when the source code is not available. The approach taken by the second group is to run the kernel and user-space applications in a virtual machine and to intercept, and respond to, the events of interest from the virtual machine monitor (VMM) [9]. Although this approach guarantees transparency and has a loose dependency on the operating system internals, it cannot be used in all the settings, since it implies that the system must be run as a guest of a virtual machine and production systems not running in virtual machines cannot be analyzed. Moreover, VMM-based solutions typically virtualize hardware devices, to allow multiple guests to share the same physical peripherals. This makes software virtualization approaches unsuitable to assist the analysis of components that need to interact directly with the underlying hardware.

In this paper we propose a framework that brings together the advantages of both approaches: it can be used on commodity production systems (i.e., off-the-shelf products, whose source code or debugging symbols are not necessarily available), since it does not require to instrument the system under test, and it is able to inspect systems running on real hardware, since it does not require an emulation container. Similarly to existing frameworks, the analyses that can be built on top of our framework include profiling and 
tracing of the kernel and user-space applications, interactive debugging, or even extension of system features. However, differently from existing frameworks, ours is fully dynamic, transparent, loosely dependent on the operating system, and fault-tolerant with respect to possible defects in the analysis code. First, our framework does not require recompilation or rebooting of the target system. Thus, it can be used to analyze any running production system, including commodity operating systems lacking native support for instrumentation and systems not running in virtual machines. Second, the framework is not invasive, since analyses can be performed on a virtually unmodified system: as explained in the paper, only a minimal driver needs to be installed and no parts of the kernel are patched in any way. Moreover, since the framework itself is not accessible from the target system, its code cannot be detected by malicious code or unwittingly influence buggy operating system components. Thus, the infrastructure can be applied to any operating system, as the majority of the facilities it supports are completely OSindependent, and the only OS-dependent functionalities are just provided to ease the development of analysis tools. Finally, the framework is fault-tolerant, as it guarantees that a defect in an analysis tool built on top of it do not damage the framework itself nor the analyzed system.

Our framework leverages hardware extensions for virtualization available on commodity x 86 CPUs $[1,15]$. Hardwaresupport for virtualization allows the development of virtual machine monitors that are very efficient, completely transparent, and non invasive to the systems running in the virtual machine. To overcome the major limitation of traditional VMM-based approaches (i.e., the impossibility to analyze productions systems not running in a virtual machine), our framework exploits a feature of the hardware that allows to install a virtual machine monitor and to migrate a running system into a virtual machine. When the analysis is completed, the original mode of operation of the system can be restored. Practically speaking, our framework is a minimalistic virtual machine monitor acting as a broker between the analyzed system and the analysis tool. The framework abstracts low-level events occurring in the analyzed system into high-level events and guarantees fault-tolerance by relying on the hardware to run the analysis tool in a isolated execution environment.

To demonstrate the potentials of our framework we have developed an interactive kernel debugger, nicknamed HyPERDBG, constructed entirely using the programming interface exposed by our infrastructure. HYPERDBG adds live and interactive debugging support to Microsoft Windows XP, so far only possible using very invasive tools, like Syser [19], or traditional VMM-based debuggers. HYPERDBG can be used to debug any component of the Windows kernel, including interrupt/exception handlers, device drivers, and even supports single instruction stepping. Being completely separated from the debuggee, HYPERDBG is transparent to the analyzed system and can be even used to analyze protected and malicious code.

In summary, the paper makes the following contributions.

1. We propose a framework to perform complex dynamic system-level analyses of commodity production systems. Compared to existing frameworks, the one we propose guarantees transparency, efficiency, and does not require the target system to be already installed on a virtual machine.
2. We implemented our framework in an experimental prototype for Microsoft Windows XP.

3. We describe the design and the implementation of $\mathrm{HY}$ PERDBG, a kernel-level interactive debugger built on top our framework.

Both the analysis framework and HYPERDBG are available at http://security.dico.unimi.it/hyperdbg/ and is released under the terms and conditions of the GPL (v3.0) license.

\section{RELATED WORK}

The framework proposed in this paper shares many similarities with frameworks and techniques extensively explored in the past. However, by exploiting recent facilities available of modern Intel x86 CPUs, our framework is able to combine and to offer simultaneously the main benefits introduced by previous research work.

\section{Dynamic Kernel Instrumentation.}

DTrace is a facility included into the Solaris kernel that allows the dynamic instrumentation of production systems [2]. The key points of DTrace are efficiency and flexibility. First, the instrumentation framework itself introduces no overhead. Second, the framework provides tens of thousands of instrumentation points, and the actions to be taken can be expressed in terms of a high-level control language, that also includes a number of mechanisms to guarantee runtime safety. Similarly, KernInst is a dynamic instrumentation framework for commodity kernels [20]. KernInst has been developed mainly to gather information about the performances of a running kernel, but it has also been employed for run-time kernel optimization. Differently from DTrace, KernInst does not provide any mechanism for runtime safety of the instrumentation routines. Unfortunately, the aforementioned approaches are not transparent, as they require direct modifications of the operating system kernel, achieved by loading a kernel-mode module. Moreover, none of them is OS-independent, and they and cannot be applied to closed-source operating systems. Our framework does not suffer these limitations since it can instrument the kernel without modifying it and does not rely on any facility offered by the kernel.

\section{Kernel-level Debugging.}

Several efforts have been made to develop efficient and reliable kernel-level debuggers. Indeed, these applications are essential for many activities, such as the development of device drivers. One of the first and most widely used kernel-level debuggers that targeted the Microsoft Windows operating system was SoftICE [18], but today the project has been discontinued. However, both commercial [19] and open-source [17] alternatives to SoftICE appeared. Modern versions of Windows already include a kernel debugging subsystem [14]. Unfortunately, to exploit the full capabilities of Microsoft's debugging infrastructure, the host being debugged must be physically linked (e.g., by means of a serial cable) with another machine. All these approaches share a common factor: to debug kernel-level code, they leverage another kernel-level module. Obviously, that is like a dog chasing its tail. The framework proposed in this paper does not require any kernel support nor to modify the kernel to add the missing support at run-time. 


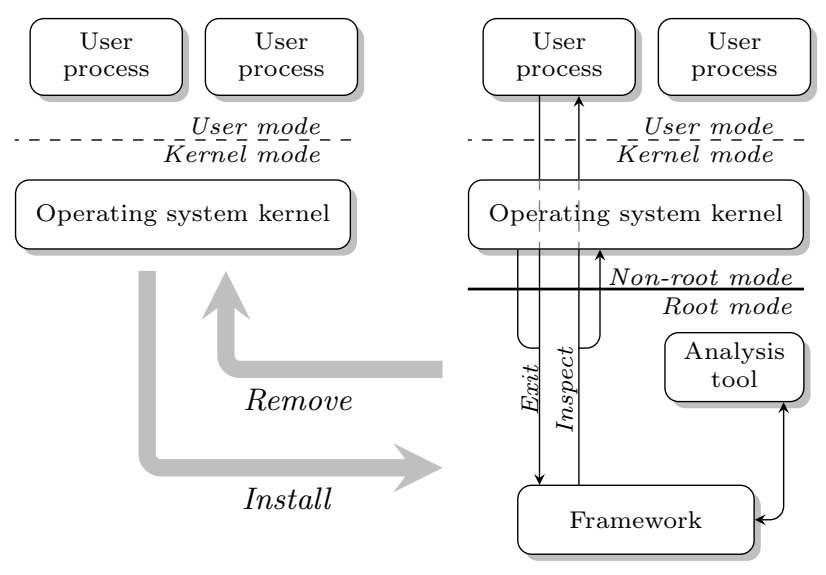

Figure 1: Overview of the framework

\section{Frameworks Based on Virtual Machines.}

Instead of relying on a kernel-level module to monitor other kernel code, an alternative approach consists of running the target code inside a virtual machine, and to perform the required analyses from the outside [9]. In [10, 22, 7] the authors propose virtual machines with execution replaying capabilities: a user can move forward and backwards through the execution history of the whole system, both for debugging and for understanding how a hacker intrusion took place. Finally, in [3] Chow et al. propose Aftersight, a system that decouples execution recording from execution trace analysis, thus reducing the overhead suffered by the system where the guest operating system is run. Nowadays, Aftersight is part of the VMware platform, and other mainstream commercial products provide similar capabilities. The framework proposed in this paper can provide these functionalities even on systems not running in any virtual machine.

\section{Aspect-oriented Programming.}

Aspect-oriented programming is a paradigm that promises to increase modularity by encapsulating cross-cutting concerns into separated code units, called "aspects", whose "advice" code is woven into the system automatically, by specifying the properties of the join-points. AspectC is an aspectoriented framework that is used to customize (at compiletime) operating system kernels [4, 12, 13]. More dynamic approaches have been proposed: for example TOSKANA provides before, after and around advices for in-kernel functions and supports the implementation of aspects themselves as dynamically exchangeable kernel modules [8]. The framework proposed in this paper allows to achieve the same goal while being transparent and fault-tolerant.

\section{OVERVIEW OF THE FRAMEWORK}

Figure 1 depicts the architecture of our framework, the installation and removal processes, and the migration of the operating system and its applications into a virtual machine. Our framework consists of a virtual machine monitor (VMM for short) that provides a programming interface for the development of system-level analysis tools. As in traditional VMM-based analysis approaches, the analysis tool is run within the VMM and thus completely transparent to guests of the virtual machine. However, compared to traditional
VMM-based ones, ours does not require the system to be already running inside any virtual machine. To achieve this goal, our framework leverages hardware extensions for virtualization available on all modern x 86 CPUs $[15,1]$ (which are unused in the majority of the deployments). In short, these extensions augment the instruction set architecture with two new modes of operation: VMX root mode and VMX nonroot mode ${ }^{1}$. These new modes of operation separate logically the virtual machine monitor from a guest without having to modify the latter. More precisely, we exploit a particular feature of these extensions that allows for late launching of VMX modes. Late launching of VMX modes permits to install a virtual machine monitor even if the system has already been bootstrapped. In other words, late launching allows to migrate (temporarily) a running operating system in a virtual machine, and to analyze and control the execution of the system from the monitor. Through the rest of the paper, we use the term "guest" to refer to the system under analysis that has been migrated into a virtual machine.

Practically speaking, the running operating system is not migrated anywhere and not touched at all. Rather, by launching VMX modes, the execution environment is extended with the two aforementioned operating modes; the running operating system is then associated with non-root mode, while the VMM is associated with root mode. Thus, in all respects, the operating system and its applications become a guest of our special virtual machine. Following the same principle, the VMM can be unloaded, and the original mode of execution of the operating system restored, by simply disabling VMX modes. After the launch of the VMX modes, the execution of the guest can continue exactly as before, even in terms of interactions with the underlying hardware devices. However, during its execution, the guest might be interrupted by an exit to root mode. Like hardware exceptions, exits are events that block the execution of the guest, switch from non-root mode to root mode, and transfer the control to the VMM. Differently from exceptions, the set of events triggering exits to root mode can be configured dynamically by the VMM. A routine of the VMM handles the exit and eventually enters non-root mode to resume the execution of the guest. Being executed at the highest privilege level, the routine handling the exit has complete read/write control of the state of the guest system (of both memory and CPU registers).

The framework itself does not perform any analysis. It is only responsible for handling a small set of exits to control all accesses to the memory management unit of the CPU, to prevent the guest from accessing the physical memory locations holding the code and the data of the framework. On the other hand, the framework provides a flexible API to develop tools to perform sophisticated analyses of both kernel and user code running in the guest. Using the functionalities provided through the API, the tool can request the framework to monitor certain events that might occur during the execution of the guest; when such events occur, it can inspect, and even manipulate, the state of the guest. The events that can be monitored include, but are not limited to, system call invocations, function calls, context switches and I/O operations. Practically speaking, events are monitored through exits to root mode. Thus, a request of the analysis tool to monitor a certain high-level event (e.g., the execu-

\footnotetext{
${ }^{1}$ VMX (non-) root mode is the terminology used by Intel; AMD adopts a different terminology.
} 


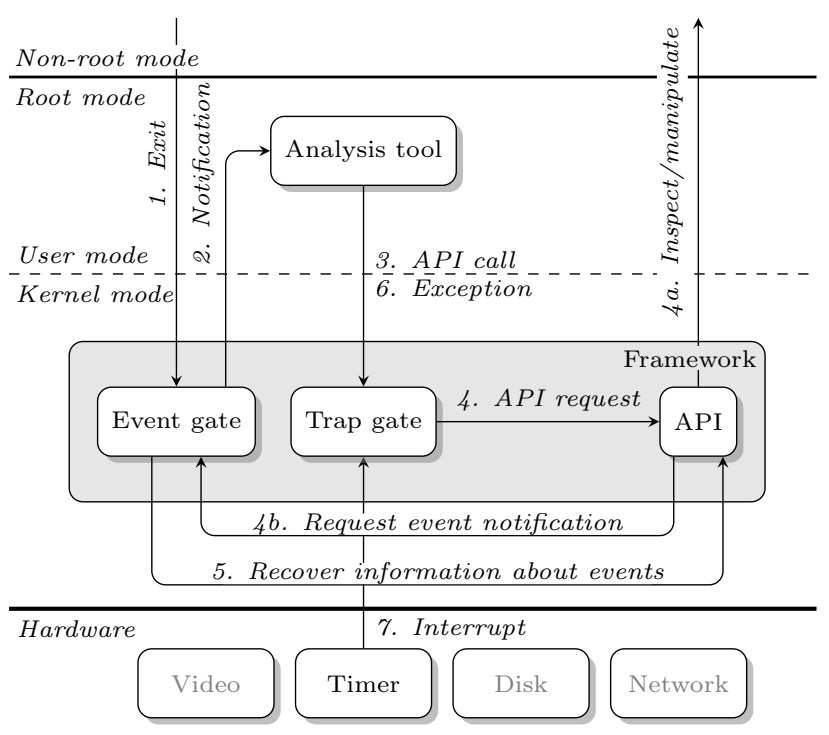

Figure 2: A close-up of the framework

tion of a system call) is translated by the API of the framework into a sequence of low-level operations that guarantee that all the occurrences of such event in the guest trigger an exit to root mode. Similarly, the framework translates the exit into a higher-level event and notifies the occurrence of the event to the analysis tool. Once notified, the tool can recover information about the event (e.g., arguments and return value of a system call), using the inspection functionalities offered by the API.

An important requirement for the analysis of production systems is that analysis tools must not interfere with the correct execution of the guest. This is particularly important for faults and deadlocks that might occur in the analysis tool. The approach we adopt is to run the tool in a less privileged execution environment, isolated from the analyzed system and from the framework. The tool can interact with the guest only through the API exposed by the framework. This approach guarantees the framework the ability to intercept any fault occurring in the tool, to mediate all accesses to the analyzed system (and to prevent write accesses), and to terminate the tool in case of deadlocks or other anomalous situations.

\section{DESIGN AND IMPLEMENTATION}

Figure 2 shows a more detailed view of the architecture of our framework. Intuitively, this architecture is very similar to that of traditional operating systems: the framework plays the role of the kernel and the analysis tool plays the role of a user-space application. As will become clear later, this architecture prevents buggy analysis tools from compromising the guest system and the framework. The separation between these two parts is made possible by the fact that, when VMX is enabled, root and non-root modes offer two fully-featured execution environments. Thus, like the guest running in non-root mode, the framework running in root mode can rely on privilege separation to isolate the analysis tool and can handle independently interrupts and exceptions that might occur while executing in root mode.

When an exit to root mode interrupts the execution of the guest, the event is delivered to the event gate (step 1 in Figure 2). The event gate is responsible for abstracting low-level events into higher-level ones, and to notify the analysis tool if the latter has requested to do so (step 2). On startup the analysis tool requests the framework to be notified of certain events (not shown in the figure). The tool can use the API provided by framework to query extra information about the event (e.g., the content of the stack location storing one of the arguments of a function). Since the tool is isolated from the framework, API functions are invoked through software interrupts. Thus, requests coming from the analysis tool are received by the trap gate (step 3 ), then forwarded to the component implementing the API (step 4). The tool can perform two types of API calls: (step 4a) to inspect or manipulate the state of the guest, and (step 4b) to control event notifications (e.g., enable or disable the notification of certain events). Note that the component implementing the API is also used by the framework itself (step 5) to recover extra information about events (e.g., the return address of a function stored in the stack). The trap gate also serves the purpose of detecting exceptions (e.g., page faults) that might occur during the execution of the analysis tool. If the trap gate intercepts an exception (step 6 ), it terminates the faulty tool and unloads the framework, to resume the normal operation mode of the system. Finally, the trap gate is also used to handle timer interrupts (step 7), that, as will be discussed in Section 4.4, are employed to enforce a time-bound on the execution of the tool.

The functionalities provided by the API of the framework can be classified into two classes: execution and I/O tracing and state inspection and manipulation. The following paragraphs describe briefly the API. More details are given in Sections 4.2 and 4.3 .

Execution and I/O tracing facilities allow a tool to intercept the occurrence in the analyzed system of certain events and certain I/O operations respectively. Table 1 reports the main types of events that can be traced. For each event, the table also reports the arguments associated to the event; arguments are information about the events most commonly used in tools. For example, the events FunctionEntry and SyscallEntry are used to trace functions and system calls respectively. The arguments associated to the FunctionEntry event are the address (or the name) of the function called, the caller and the return address. Another example is the ProcessSwitch event that can be used to trace context switches between processes (not threads). From the point of view of the analysis tool all the events are handled in the same way: the tool can subscribe to any event and, when the event occurs, can inspect its arguments and take the proper actions. However, at the framework-level, certain events are different from other ones. Indeed, some of them (e.g., context switches between processes) can be traced directly by the hardware. That is, the event triggering the exit corresponds exactly to the event being traced. Other events instead (e.g., function calls and returns) cannot be traced directly by the hardware. In all these cases the framework relies on other low-level events to trace the execution and then abstract exiting low-level events into higher-level ones, meaningful for the analysis tool.

Arguments can optionally be used as conditions, to limit the tracing to a subset of all the events. Conditions on events serve two purposes. First, conditions allow to simplify the analysis tools, since events that do not match the requested conditions are discarded by the framework and thus do not 


\begin{tabular}{lll}
\hline Event & Description & Arguments \\
\hline ProcessSwitch & Context (process) switch & - \\
Exception & Execution & Exception vector, faulty instruction, error code \\
Interrupt & Hardware or software interrupt & Interrupt vector, requesting instruction \\
BreakpointHit & Execution breakpoint & Breakpoint address \\
WatchpointHit & Watchpoint on data read/write & Watchpoint address, access type, hitting instruction \\
FunctionEntry & Function call & Function name/address, caller/return address \\
FunctionExit & Return from function & Function name/address, return address \\
SyscallEntry & System call invocation & System call number, caller/return address \\
SyscallExit & Return from system call & System call number, return address \\
IODperationPort & I/O operation throught hardware port & Port number, access type \\
IOOperationMmap & Memory-mapped I/O operation & Memory address, access type \\
\hline
\end{tabular}

Table 1: Events traceable using our framework and corresponding arguments (the argument that represents the current process is omitted, as it is common to all the events)

need to be handled by the tool. Second, some conditions allow preemptive filtering of the events. In other words, the framework configures a priori which events trigger an exit, instead of filtering out exits caused by uninteresting events. For example, in the case of the IOOperationPort event, preemptive filtering means to configure the CPU such that only I/O operations involving a specific I/O port trigger an exit. This feature is very important to minimize the number of exits and thus the overall overhead.

State inspection and manipulation primitives can be used by the tool to access the state of the guest, in order to extract more detailed information about events or other data useful for the analysis. For example, these primitives allow to extract the arguments of an invoked function, or to inspect the internal structures of the guest operating system. Note that, by default, write access to guest state is not granted to a tool. If necessary, such permission can be enabled at compile-time. Obviously, in this case the framework cannot protect the state of the guest from dangerous modifications.

\subsection{Framework and Analysis Tool Loading}

The framework and the analysis tool are loaded by a minimal kernel driver. This is unavoidable since the operations we need to perform to load the framework require maximum privileges and can be performed only by the kernel of the operating system. The driver, however, is indeed very simple and we put extreme care in avoiding any interference with the kernel. Moreover, since once loaded the framework is completely invisible to the system, we unload the driver immediately as soon as the framework has been installed.

When VMX modes are enabled, a special VMX data structure (VMCS in Intel terminology) is made accessible initially to the loader, and subsequently, when the loading is completed, only to the framework. This data structure stores the host state, guest state, and the execution control fields. The host state stores the state of the processor that is loaded on exits to root mode, and consists of the state of all the registers of the CPU (except for general purpose registers). Similarly, the guest state stores the state of the processor that is loaded on entries to non-root mode. The guest state is updated automatically at every exit, such that the subsequent entry to non-root mode will resume the execution from the same point. The execution control fields allow a fine-grained specification of which events should trigger an exit to root mode.

The task of the loader is to enable VMX modes and to configure the VMX data structure such that the execution of the operating system and user-space applications continue to run in non-root mode, while the framework and the analysis tool are executed in root mode. Moreover, the loader has to configure the CPU such that all the events necessary for the tool to trace the execution of the system trigger exits to root mode. When the initialization is completed, the driver unloads itself and resumes the execution of the system.

\section{Guest State Configuration.}

The guest state is initialized to the current state of the system. In this way, when the virtual machine is launched and execution enters non-root mode, the guest operating system will resume its execution as if nothing happened. A tricky problem when initializing non-root mode concerns the management of the memory. More precisely, we must prevent the newly created guest to use and access the physical memory frames allocated to the framework and to the tool. Otherwise, the guest could detect and even corrupt the framework. Most recent CPUs provide hardware facilities for memory virtualization (e.g., Intel Extended Page Table extension). If these facilities are not available, memory virtualization must be implemented entirely via software. Briefly, software memory virtualization consists of intercepting all guest operations to manipulate the page table (the data structure the CPU uses for virtual-to-physical address translation) and in ensuring that none of the physical frames allocated to the framework and to the analysis tool are mapped into the guest. In case the guest tries to map a reserved physical frame, the framework assigns the guest a different one and masquerades the difference.

\section{Host State Configuration.}

The host state is initialized as follows. The CPU is configured to use, when in root mode, a dedicated address space and a dedicated interrupt descriptor table (IDT). This configuration simplifies the separation of the analyzed system from the framework and allows to detect and handle interrupts and exceptions that occur in root mode. Differently from the address of the entry point of non-root mode, which is updated at every exit to allow to resume execution of the guest from where it was interrupted, the address of the entry point of root mode is fixed. The entry point is set to the address of the routine that takes care of dispatching an exit event to the appropriate handler and that in turn might notify the analysis tool (i.e., the entry point of the event gate).

\section{Execution Control Fields Configuration.}

To reduce the run-time overhead suffered by the guest 


\begin{tabular}{|c|c|c|}
\hline Event & Exit cause & $\begin{array}{c}\text { Native } \\
\text { exit }\end{array}$ \\
\hline ProcessSwitch & Change of page table address & $\sqrt{ }$ \\
\hline Exception & Exception & $\sqrt{ }$ \\
\hline Interrupt & Interrupt & $\sqrt{ }$ \\
\hline BreakpointHit & Debug except. / Page fault except. & \\
\hline WatchpointHit & Page fault except. & \\
\hline FunctionEntry & Breakpoint on function entry point & \\
\hline FunctionExit & Breakpoint on return address & \\
\hline SyscallEntry & Breakpoint on syscall entry point & \\
\hline SyscallExit & Breakpoint on return address & \\
\hline I0OperationPort & Port read/write & $\sqrt{ }$ \\
\hline IOOperationMmap & Watchpoint on device memory & \\
\hline
\end{tabular}

Table 2: Techniques for tracing events

system, the execution control fields are configured to minimize the number of events that trigger an exit to root mode. When the tool is initialized, it specifies which events must be intercepted. Subsequently, in response to the invocation of API functions, the configuration of the execution control fields can be altered to intercept additional events or to ignore other ones.

\subsection{Execution Tracing}

Table 2 describes the technique used to trace all the events currently supported by the framework. Low-level events (those with a mark in the last column) correspond directly to exits to root mode (e.g., Exception). Other events are traced through the aforementioned ones (e.g., BreakpointHit), and others again are traced through the latter (e.g., FunctionEntry).

Events that can be traced directly through the hardware are process switches, exceptions, interrupts, and port-based I/O operations. All these events exit conditionally: they exit to root mode only when requested and can have optional exit conditions to limit exits to particular situations. The remaining of this section presents how we developed the primitives for tracing higher-level events starting from the aforementioned low-level ones.

Breakpoints and watchpoints are two of the most complicated events to implement. Modern CPUs provide hardware facilities to realize efficient and transparent breakpoints and watchpoints. Unfortunately, hardware-assisted breakpoints and watchpoints are limited in number (only 4) and shared between non-root and root mode. Therefore, they cannot be used simultaneously by the analyzed system and by the framework. The solution we adopt to allow an arbitrary number of breakpoints is to use software breakpoints. A software breakpoint is a one-byte instruction that triggers a breakpoint exception when executed. Software breakpoints are enabled by replacing the byte at the address on which we want the breakpoint with the aforementioned instruction. When the breakpoint is hit, the original byte is restored and the event is notified to the tool. If the breakpoint is not persistent the execution of the system is resumed. Otherwise the instruction is emulated and then the breakpoint is set again. Clearly, this approach to breakpoints is not transparent for the analyzed system. However, it is very efficient. An alternative and transparent approach is to use the same technique we use for watchpoints, as described in the next paragraph. Our framework supports both approaches.

The approach used in our framework to implement software watchpoints is based on protecting the memory locations from any access via hardware (or just from write ac- cesses, depending on the type of watchpoint), such that any access results in an exception [21]. More precisely, since the finest level of protection offered by the hardware is at the page level, we mark the page containing the address on which we want to set the watchpoint as "non-present". Any future access to this page will result in a page fault exception that will be intercepted by our framework. The framework analyzes the exception and checks whether the accessed address corresponds to the address with the watchpoint. If the watchpoint is hit, the framework delivers the event to the analysis tool, otherwise it emulates the instruction, and then resumes the normal execution of the guest. Emulation is necessary to execute the faulty instruction manually. Indeed, to prevent a second fault, the original permission of the memory page accessed by the instruction must be restored before executing the faulty instruction. After the execution of the instruction, the page must be marked again as "non-present" to catch future accesses.

Other higher-level events, such as function and system call entries and exits, are traced through breakpoints. When the analysis tool requests the framework to monitor a certain function, the framework sets a breakpoint on the address of the entry point of the function. Later, when a breakpoint is hit, the framework checks whether the hit breakpoint corresponds to a function entry point and, if so, it delivers the appropriate event (i.e., FunctionEntry) to the analysis tool. Function exits, instead, are traced by setting a breakpoint on the return address. The framework discovers the return address by setting a breakpoint on the function entry and by inspecting the stack frame of the function when the breakpoint on the entry point is hit. A similar approach is used for tracing system calls entries and exits.

The approach for tracing function calls and returns just described allows to trace specific functions, whose names or addresses are supplied by the tool. The tracing of all function calls and returns is instead more complicated because it is not possible to know a priori the addresses of all functions' entry points. The solution in this case is to perform a static analysis to identify the addresses of all functions' entry points (e.g., by recognizing function prologues). This feature is still not available in our current implementation of the framework. Nevertheless, if needed, the static analysis could be performed directly in the tool. The tracing of all system calls is instead much easier, since they are all invoked through a common gate. The solution we adopt is to put a breakpoint on the entry point of the system call gate [6].

Beside execution tracing facilities, the framework also exposes to analysis tools the possibility of intercepting I/O operations with hardware peripherals. Software can interact with hardware devices through hardware I/O ports, or it can leverage memory-mapped I/O. In the first case, VMX allows to intercept the operation without any effort: the framework simply configures the execution control fields such that all the interactions with the specific hardware ports trigger an exit to root mode; when such an exit occurs, the framework notifies the tool by means of a I00perationPort event. However, for performance reasons, modern peripherals typically resort to memory-mapped I/O. In this case, read and write operations do not involve any hardware port, as they are performed directly on memory. To intercept such operations we set a watchpoint on the appropriate memory region. Thus, when an access to it is detected, the framework delivers a I00perationMmap event to the tool. 


\subsection{State Inspection and Manipulation}

Several situations require to access the state of the guest system in order to inspect, and optionally manipulate, both the registers of the CPU and the memory. As an example, the framework could need to read the return address of a function from the stack, to access the parameters of a system call from the processor registers, or to insert a breakpoint into the address space of a particular process. Similarly, the analysis tool might need to extract data from the memory of the guest.

The inspection and manipulation of $\mathrm{CPU}$ registers is a straightforward activity. These information are saved during an exit and restored before an entry. Thus, the inspection and manipulation of registers merely consists of reading or writing the VMX guest state (or the memory of the framework, depending on the type of register).

Inspection and manipulation of memory locations is much more complex. When paging is enabled, virtual addresses are translated by the hardware into physical addresses according to the content of the page table and direct physical addressing is not possible. Each process has its own page table; therefore, different processes have different virtual-tophysical mappings and a process cannot access the memory of the others. The framework is isolated from the guest using the same approach and thus it has its own page table and its own mapping. Consequently, the framework cannot directly access memory locations of guest processes. Moreover, inspection is complicated by the fact that page tables cannot be traversed via software (but only via hardware): the page table is a multilevel table and pointers to lower levels are physical. To overcome this problem we have developed a specific, OS-independent, algorithm that allows to access an arbitrary virtual memory location of an arbitrary process. The core of the algorithm is a primitive that allows to access arbitrary physical memory locations. This is accomplished by mapping a given physical address $p$ to an unused virtual address $v$ in the page table of the framework, and subsequently by accessing $v$. Then, using this primitive, the algorithm can traverse the page table of a process of the guest via software by iteratively mapping the physical addresses stored in the table.

The framework exposes memory inspection and manipulation facilities, based on the aforementioned algorithm, to the analysis tools through two API functions: GuestRead $(p, a,-$ $n$ ) and GuestWrite $(p, a, \operatorname{dat} a)$. The former reads $n$ bytes starting from virtual address $a$ of process $p$; the latter writes the content of buffer data into the address space of process $p$, starting from virtual address $a$. By default, to preserve the integrity of the guest, all GuestWrite operations are forbidden. On top of this functions we have built higher-level ones that facilitates the extraction of functions' arguments, null terminated strings, and to disassemble code.

\subsection{Tool Isolation}

To be able to use our infrastructure on a production system, it is essential to guarantee that any defect in the analysis tool will not affect the stability of the analyzed system and of the framework. At this aim, the framework controls the execution of the analysis tool and, if any anomalous behavior is observed, the whole infrastructure is automatically unloaded.

As we outlined at the beginning of this section, even if the analysis tool is executed in VMX root mode, it is still constrained into a less privileged execution mode than the framework. Thus, any operation the tool performs on the guest must be mediated by the framework. This is exactly what happens in traditional operating systems: a user-mode process cannot access directly the resources of the operating system, nor those of other user-mode processes, and any action it performs outside its address space must be mediated by the kernel. Similarly in our context, to perform an operation on the guest system, the tool must use the programming interface offered by the framework.

In the default configuration, the framework does not allow a tool to access in write-mode to the state of the guest. However, there is still the possibility that the execution of an instruction of the tool raises an unexpected exception (e.g., a page fault on memory access, or a general protection fault). When such an event occurs, the framework has no way to handle the anomalous situation and to allow the tool to continue its execution. The only viable approach that also preserves the integrity of the guest system is to terminate the analysis tool and to remove the framework. At this aim, the solution we adopt is to intercept unexpected exceptions through the custom interrupt descriptor table (IDT) installed when launching VMX modes. The IDT receives the trap, and delivers it to the trap gate that eventually unloads the framework. Another problem that might arise with a buggy analysis tool is non-termination: if the analysis tool entered an infinite loop, the guest system would never be resumed. To prevent this problem we added to the framework a minimalistic watchdog and set a time limit on the execution of the tool. The limit is not on the whole execution time of the tool, but rather on the execution time to handle an event. Thus, the analysis tool could potentially be run forever, but with the guarantee that the execution of the analyzed system will be resumed within the specified time limit. At this aim, before delivering an event to the analysis tool, the framework resets a timer. Then, while the tool handles the event, the framework periodically regains the control of the execution and checks whether the time limit has been exceeded. To do that the framework registers, in the IDT, a custom interrupt handler to handle timer interrupts and programs the interrupt controller to deliver only timer interrupts (that is necessary to prevent the framework to consume interrupts for all the other devices). Before returning to non-root mode, the framework reprograms the interrupt controller to deliver all the interrupts to the analyzed system.

\subsection{OS-dependent Interface}

Our framework provides a general programming interface completely independent from the operating system running inside the guest. However, in many cases some OS-specific facilities can ease the analysis of the guest. As an example, the only OS-independent manner to identify a process is by means of the base address of its page table (typically stored inside the cr3 CPU register). However, it is quite awkward to refer to processes using page table base addresses, and it is more natural to identify a process through its process id (PID) or through the name of the application it executes.

The OS-dependent interface we provide leverages virtual machine introspection techniques [9] to analyze the internal structures of the guest operating system to translate OS-independent information (e.g., process with page table base address 0x13cdc000) into something more user-friendly 


\begin{tabular}{|c|c|}
\hline Name & Description \\
\hline GetFuncAddr $(n)$ & Return the address of the function $n$ \\
\hline GetFuncName $(a)$ & Return the name of the function at address $a$ \\
\hline $\operatorname{GetProcName}(p)$ & $\begin{array}{l}\text { Get the name of process with page directory } \\
\text { base address } p\end{array}$ \\
\hline $\operatorname{GetProcPID}(p)$ & $\begin{array}{l}\text { Get the PID of process with page directory } \\
\text { base address } p\end{array}$ \\
\hline $\operatorname{GetProcLibs}(p)$ & $\begin{array}{l}\text { Enumerate the dynamically linked libraries } \\
\text { loaded into process } p\end{array}$ \\
\hline $\operatorname{GetProcStack}(p)$ & Get the stack base for process $p$ \\
\hline $\operatorname{GetProcHeap}(p)$ & Get the heap base for process $p$ \\
\hline GetProcList() & Enumerate processes \\
\hline GetDriverList() & Enumerate device drivers \\
\hline
\end{tabular}

Table 3: OS-dependent API

(e.g., process notepad.exe). Moreover, using debugging symbols, the framework allows to resolve symbols' names and addresses (e.g., functions and global variables). In this way, a tool can ask to interrupt the execution of the guest when function NtCreateFile is invoked, instead of referencing the function through its address. Similarly, when a function is invoked, it is possible to inspect its call-stack and to resolve the name of the caller functions and even recover the libraries to which the various functions belong to. Some of the OS-dependent functionalities provided are summarized in Table 3.

In case the guest operating system is not supported, the OS-dependent module is disabled, and only OS-independent functionalities are available. Our current implementation offers an OS-dependent interface only for the Windows XP operating system.

\section{APPLICATIONS}

In this section we present HYPERDBG, an interactive kernel debugger for Microsoft Windows XP we built on top of our framework. In our strive to contribute to the open source community, we released the code of HYPERDBG, along with the code of the framework, under the GPL (v3.0) license. The code is available at the following address:

\section{http://security.dico.unimi.it/hyperdbg/}

The section also discusses other possible applications that could be constructed using our framework.

\subsection{HyperDbg}

HyPERDBG is an interactive kernel debugger we developed on top of our analysis framework. It offers all the features commonly found in kernel-level debuggers but, being completely run in VMX root mode, it is OS-independent and grants complete transparency to the guest operating system and its applications. The debugger provides a simple graphical user interface to ease the interaction with the user. This interface is activated in two circumstances: (I) when the user presses a special hot-key or (II) when the debugger receives the notification for an event that requires the attention of the user (e.g., when a breakpoint is hit). From this interface the user interacts with the debugger and can perform several operations, including setting breakpoints and watchpoints, tracing functions and system calls, and inspecting and manipulating the state of the guest (since all interactive debuggers allow to modify the state of the debuggee, we decided to enable write access to the guest as well).

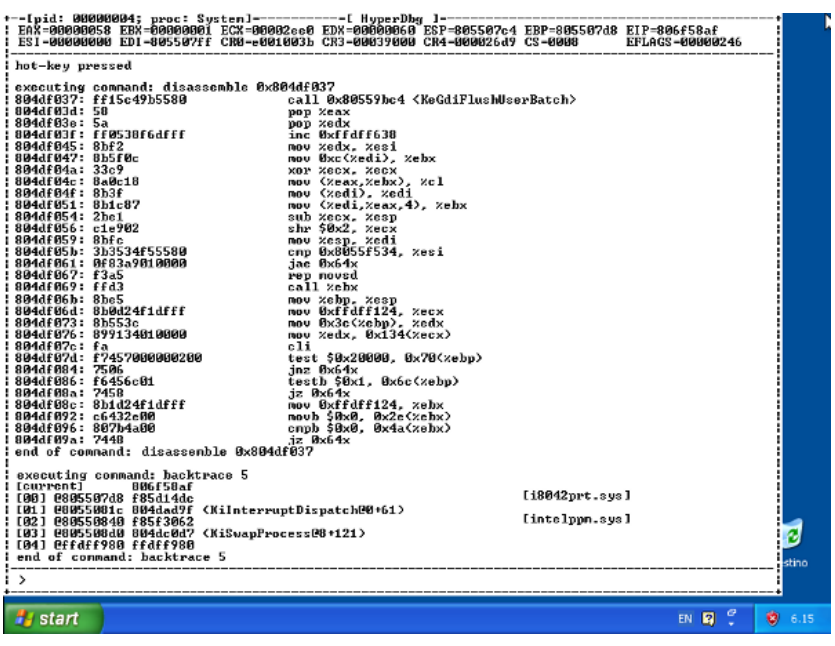

Figure 3: HyperDbg in action

Figure 3 shows HyPERDBG in action ${ }^{2}$. In particular, the figure shows the debugger notifying the event that interrupted the execution of the analyzed system, displaying a fragment of the code of the process currently running in the analyzed system and displaying a "backtrace" of the function calls that are currently active. Additionally, the debugger displays information about the status of the registers at the time the event occurred (in the case of the figure the event is the pressure of the hot-key). To facilitate the analysis, the debugger leverages OS-dependent information. For example, the screenshot in Figure 3 shows that the debugger resolved the ID and the name of the process in a MS Windows $\mathrm{XP}$ guest, by knowing how the process table is managed by the operating system.

It is worth pointing out that HYPERDBG can be used to debug any piece of code of the guest system, including critical components such as the process scheduler, or interrupt and exception handlers. Indeed, Figure 3 shows that the guest operating system has been stopped while executing the PS/2 keyboard/mouse driver (i8042prt.sys). Thanks to the fact that the framework on which the debugger is built on is completely transparent to the analyzed system, the user can use the keyboard to interact with the debugger even though the keyboard driver of the guest is being debugged.

HYPERDBG consists of less than 1600 lines of code: $\sim 25 \%$ of the code implements the graphical interface, $\sim 23 \%$ of the code provides the facilities required for keyboard-based user interaction, and the remaining $\sim 52 \%$ is responsible for handling events and for all the other interactions with the framework. Note that certain functionalities (e.g., disassembling a code region) are implemented directly in the framework since, most likely, they will be used for other types of analysis as well. The framework is about four times bigger than the debugger (without considering the disassembly module embedded in the framework, as it is based on an off-the-shelf disassembler). We believe these numbers are very significant. The number of lines of code we had to write to implement HYPERDBG clearly witnesses that com-

\footnotetext{
${ }^{2}$ The screenshot was taken using our development environment based on an Intel x86 emulator supporting extensions for virtualization (i.e., BOCHS).
} 
plex analysis tools like an interactive kernel debugger are straightforward to implement using our framework.

The remaining of this section describes how we used the facilities of the framework to implement the user interface and the component to receive commands from the user.

\section{User Interface.}

Although the graphical user interface of the debugger is rough, its implementation is very challenging. The reason of the complexity is the fact that we cannot rely on any high-level graphical facility available in the analyzed system to render the interface. Such approach would be too OSdepended and not transparent at all. The lack of graphical primitives obliged us to interact directly with the video card. The video memory is mapped at a fixed address in the guest and thus unmodified inspection and manipulation API (i.e., GuestRead and GuestWrite) can be used by the debugger to render the interface. Note that this approach is not dependent on the OS nor on the hardware. We developed a small video library that provides basic graphical functionalities and translates our requests into data that are written directly in the memory of the video card. Before rendering the graphical interface to the screen, the debugger backups the content of the video memory and restores the content right before resuming the execution of the analyzed system.

\section{User Interaction.}

User interaction is keyboard-based. When in non-root mode, the user can switch into HYPERDBG by pressing a hot-key. Then, in root mode the user can control the debugger. For these reasons, HyPERDBG must be able to intercept keystrokes both in root and non-root mode. To intercept keystrokes in non-root mode we monitor all the read operations from the hardware I/O port devoted to the keyboard. In other words, HYPERDBG registers to the core for all the IOOperationPort events that satisfy the event condition port $=$ KEYBOARD_PORT \&\& access=read. When such operation is detected, HYPERDBG checks whether the key pressed corresponds to the hot-key that enables the debugger. If the key pressed matches the hot-key the debugger pops up the graphical interface and waits for commands. Otherwise, the debugger passes the keystroke to the analyzed system such that the latter will continue its execution as if the keystroke were read directly from the keyboard. Keyboard handling in root mode is done by polling the keyboard hardware I/O port. Since direct access to I/O ports is not permitted to any analysis tool, the debugger relies on a API function exported by the framework which mediates all accesses to I/O ports and allows (if the permission is granted at compile time) certain analysis tools to read data from certain I/O ports.

\subsection{Other Possible Uses of the Framework}

HYPERDBG demonstrates that our framework is very versatile and that enables new opportunities for dynamic analysis and we will explore in our future research.

An interesting extension of HYPERDBG will be the support for kernel-level omniscent debugging. Omniscent debugging allows developers to inspect the status of their programs in past execution instants, in order to detect the cause of a failure without the need to run the target program multiple times [16]. HyperDBG could be extended to allow a user to record and inspect the values a memory location stored during the time, and the exceptions and interrupts occurred. Such a feature would ease a user to discover when a memory location of the kernel gets corrupted and which instruction is responsible for the corruption. Moreover, the ability to log asynchronous events, such as interrupts, would allow to spot defects connected to non-deterministic behaviors of the analyzed system. Our framework already offers all the necessary facilities for this kind of debugging: exception and interrupts can be traced natively by the framework and memory accesses can be traced using watchpoints.

Another interesting application of our framework will be dynamic aspect-oriented programming of operating system kernels. As discussed in Section 2, several approaches have been proposed to apply AOP to kernels. The main advantage offered by our framework over the approaches proposed so far is that it does not require any modification of the source code of the kernel, nor any modification of the image in memory of the kernel. Moreover, our framework protects the running kernel from defects in the woven code. One approach to facilitate the use of such technology would be to provide programmers a source-to-source translator, to translate aspect oriented code written in languages like AspectC [5] into $\mathrm{C}$ code that uses the API offered by our framework. In particular, the translator would be responsible for translating pointcuts into API calls to trace the corresponding events, using advices as events handlers, and for translating all pointer dereferences into calls to inspection API to read the memory of the guest.

\section{CONCLUSIONS}

We proposed a framework to perform complex run-time analyses of both system- and user-level code on commodity production systems. The framework exposes an API that eases the development of analysis tools on its top. The approach we described leverages hardware extensions for virtualization available on modern processors to overcome the limitations that affect existing approaches for the analysis of system-level code. In particular, the solution we proposed does not require to recompile or reboot the target system, it is not invasive, it is almost completely OS-independent, and it guarantees that a defect in an analysis tool cannot damage the framework itself nor the analyzed system. To demonstrate its potentials, we developed HYPERDBG, an interactive kernel-level debugger for Microsoft Windows XP. HyPERDBG and the framework have been released as an open source package.

\section{Acknowledgments}

This research has been partially funded by the European Commission, Program IDEAS-ERC, Project 227977 SMSCom and by the Italian Ministry of Education, Universities and Research, Program PRIN-2008.

\section{REFERENCES}

[1] AMD, Inc. AMD virtualization. http://www.amd.com/us/products/technologies/ virtualization/Pages/virtualization.aspx.

[2] B. Cantrill, M. W. Shapiro, and A. H. Leventhal. Dynamic Instrumentation of Production Systems. In Proceedings of USENIX Annual Technical Conference, pages 15-28, June 2004. 
[3] J. Chow, T. Garfinkel, and P. Chen. Decoupling Dynamic Program Analysis from Execution in Virtual Environments. In Proceedings of USENIX Annual Technical Conference, pages 1-14, June 2008.

[4] Y. Coady, G. Kiczales, M. Feeley, and G. Smolyn. Using AspectC to improve the modularity of path-specific customization in operating system code. In Proceedings of the 8th European Software Engineering Conference, pages 88-98, 2001.

[5] Y. Coady, G. Kiczales, M. J. Feeley, N. C. Hutchinson, and J. S. Ong. Structuring Operating System Aspects. Communications of the ACM, 44(10):79-82, 2001.

[6] A. Dinaburg, P. Royal, M. Sharif, and W. Lee. Ether: Malware Analysis via Hardware Virtualization Extensions. In Proceedings of the 15th ACM Conference on Computer and Communications Security, pages 51-62. ACM, 2008.

[7] G. W. Dunlap, S. T. King, S. Cinar, M. A. Basrai, and P. M. Chen. ReVirt: Enabling Intrusion Analysis Through Virtual-Machine Logging and Replay. In OSDI '02: 5th Symposium on Operating Systems Design and Implementations, December 2002.

[8] M. Engel and B. Freisleben. TOSKANA: A toolkit for operating system kernel aspects. Transactions on Aspect-Oriented Software Development II, 4242:182-226, 2006.

[9] T. Garfinkel and M. Rosenblum. A Virtual Machine Introspection Based Architecture for Intrusion Detection. In Proceedings of the Symposium on Network and Distributed Systems Security, San Diego, CA, Feb. 2003.

[10] S. T. King, G. W. Dunlap, and P. M. Chen. Debugging operating systems with time-traveling virtual machines. In Proceedings of USENIX Annual Technical Conference, pages 71-84, April 2005.

[11] Linux Trace Toolkit. http://lttng.org/.

[12] D. Mahrenholz, O. Spinczyk, A. Gal, and W. Schröder-Preikschat. An Aspect-Oriented Implementation of Interrupt Synchronization in the PURE Operating System Family. In Proceedings of the 5th ECOOP Workshop on Object Orientation and Operating Systems, pages 49-54, June 2002.

[13] D. Mahrenholz, O. Spinczyk, and

W. Schröder-Preikschat. Program Instrumentation for Debugging and Monitoring with AspectC++. In Symposium on Object-Oriented Real-Time Distributed Computing, pages 249-256, April 2002.

[14] Microsoft Corporation. Debugging Tools for Windows. http://www.microsoft.com/whdc/devtools/ debugging/default.mspx.

[15] G. Neiger, A. Santoni, F. Leung, D. Rodgers, and R. Uhlig. Intel Virtualization Technology: Hardware Support for Efficient Processor Virtualization. Intel Technology Journal, 10(3):167-177, August 2006.

[16] G. Pothier and E. Tanter. Back to the future: Omniscient debugging. IEEE Software, 26:78-85, 2009.

[17] Rasta ring 0 debugger. http://rrod.droids-corp.org/.

[18] SoftICE. http://en.wikipedia.org/wiki/SoftICE.

[19] Syser Kernel Debugger. http://www. sysersoft.com/.

[20] A. Tamches. Fine-Grained Dynamic Instrumentation of Commodity Operating System Kernels. PhD thesis, University of Wisconsin-Madison, 2001.

[21] A. Vasudevan and R. Yerraballi. Stealth Breakpoints. In 21st Annual Computer Security Applications Conference, pages 381-392, 2005.

[22] M. Xu, V. Malyugin, J. Sheldon, G. Venkitachalam, and B. Weissman. ReTrace: Collecting execution trace with virtual machine deterministic replay. In Proceedings of the 3rd Annual Workshop on Modeling, Benchmarking and Simulation, 2007. 


\title{
Dynamic and Transparent Analysis of Commodity Production Systems
}

\author{
Aristide Fattori $^{\dagger} \quad$ Roberto Paleari ${ }^{\dagger}$ \\ \{aristide,roberto\}@security.dico.unimi.it \\ Lorenzo Martignoni ${ }^{\ddagger}$ \\ Mattia Monga \\ lorenzo.martignoni@uniud.it \\ mattia.monga@unimi.it
}

\author{
Dip. di Informatica e Comunicazione ${ }^{\dagger}$ \\ Università degli Studi di Milano \\ I-20135 Milan, Italy
}

\author{
Dip. di Fisica $\ddagger$ \\ Università degli Studi di Udine \\ I-33100 Udine, Italy
}

\begin{abstract}
We propose a framework that provides a programming interface to perform complex dynamic system-level analyses of deployed production systems. By leveraging hardware support for virtualization available nowadays on all commodity machines, our framework is completely transparent to the system under analysis and it guarantees isolation of the analysis tools running on its top. Thus, the internals of the kernel of the running system needs not to be modified and the whole platform runs unaware of the framework. Moreover, errors in the analysis tools do not affect the running system and the framework. This is accomplished by installing a minimalistic virtual machine monitor and migrating the system, as it runs, into a virtual machine. In order to demonstrate the potentials of our framework we developed an interactive kernel debugger, nicknamed HYPERDBG. HyperDbG can be used to debug any critical kernel component, and even to single step the execution of exception and interrupt handlers.
\end{abstract}

\section{Categories and Subject Descriptors}

D.2.5 [Software Engineering]: Testing and DebuggingDebugging aids, Monitors, Tracing; D.4.9 [Operating Systems]: Systems Programs and Utilities

\section{General Terms}

Verification

\section{Keywords}

hardware virtualization, debugging, system analysis

\section{INTRODUCTION}

Operating systems are peculiar and very complex pieces of software whose internals are critically vital for a system: a failure, or a bottleneck, in any of their parts can lead to

Permission to make digital or hard copies of all or part of this work for personal or classroom use is granted without fee provided that copies are not made or distributed for profit or commercial advantage and that copies bear this notice and the full citation on the first page. To copy otherwise, to republish, to post on servers or to redistribute to lists, requires prior specific permission and/or a fee.

ASE'10, September 20-24, 2010, Antwerp, Belgium.

Copyright 2010 ACM 978-1-4503-0116-9/10/09 ...\$10.00. catastrophic consequences. Therefore, special care is needed to develop, analyze, test, and profile them. To simplify their task, developers and analysts rely on a large variety of tools and analysis techniques. Some of them are specific for studying static properties of the operating system, while others are more specific for studying dynamic properties. In particular, the latter class of tools and techniques is nowadays very popular among kernel developers and analysts because it allows them to collect the information very quickly, while hiding many of the intricacies of the kernel, and can even be used on running production systems.

Existing approaches for dynamic analysis of operating systems (e.g., debugging, profiling, and tracing) can be roughly classified in two groups: kernel-based and VMM-based. The approach taken by the first group is to include some component into the kernel in order to intercept all the events of interest (e.g., the creation of a new process, the execution of a system call, and the execution of a kernel function) and to execute a specific action when such events occur [?, ?, ?, ?, ?]. This solution requires the installation of specific hooks in the kernel to monitor run-time events and it might be very difficult to apply to operating systems that do not natively offer facilities for dynamic analysis, especially when the source code is not available. The approach taken by the second group is to run the kernel and user-space applications in a virtual machine and to intercept, and respond to, the events of interest from the virtual machine monitor (VMM) [?]. Although this approach guarantees transparency and has a loose dependency on the operating system internals, it cannot be used in all the settings, since it implies that the system must be run as a guest of a virtual machine and production systems not running in virtual machines cannot be analyzed. Moreover, VMM-based solutions typically virtualize hardware devices, to allow multiple guests to share the same physical peripherals. This makes software virtualization approaches unsuitable to assist the analysis of components that need to interact directly with the underlying hardware.

In this paper we propose a framework that brings together the advantages of both approaches: it can be used on commodity production systems (i.e., off-the-shelf products, whose source code or debugging symbols are not necessarily available), since it does not require to instrument the system under test, and it is able to inspect systems running on real hardware, since it does not require an emulation container. Similarly to existing frameworks, the analyses that can be built on top of our framework include profiling and 
tracing of the kernel and user-space applications, interactive debugging, or even extension of system features. However, differently from existing frameworks, ours is fully dynamic, transparent, loosely dependent on the operating system, and fault-tolerant with respect to possible defects in the analysis code. First, our framework does not require recompilation or rebooting of the target system. Thus, it can be used to analyze any running production system, including commodity operating systems lacking native support for instrumentation and systems not running in virtual machines. Second, the framework is not invasive, since analyses can be performed on a virtually unmodified system: as explained in the paper, only a minimal driver needs to be installed and no parts of the kernel are patched in any way. Moreover, since the framework itself is not accessible from the target system, its code cannot be detected by malicious code or unwittingly influence buggy operating system components. Thus, the infrastructure can be applied to any operating system, as the majority of the facilities it supports are completely OSindependent, and the only OS-dependent functionalities are just provided to ease the development of analysis tools. Finally, the framework is fault-tolerant, as it guarantees that a defect in an analysis tool built on top of it do not damage the framework itself nor the analyzed system.

Our framework leverages hardware extensions for virtualization available on commodity x86 CPUs [?, ?]. Hardwaresupport for virtualization allows the development of virtual machine monitors that are very efficient, completely transparent, and non invasive to the systems running in the virtual machine. To overcome the major limitation of traditional VMM-based approaches (i.e., the impossibility to analyze productions systems not running in a virtual machine), our framework exploits a feature of the hardware that allows to install a virtual machine monitor and to migrate a running system into a virtual machine. When the analysis is completed, the original mode of operation of the system can be restored. Practically speaking, our framework is a minimalistic virtual machine monitor acting as a broker between the analyzed system and the analysis tool. The framework abstracts low-level events occurring in the analyzed system into high-level events and guarantees fault-tolerance by relying on the hardware to run the analysis tool in a isolated execution environment.

To demonstrate the potentials of our framework we have developed an interactive kernel debugger, nicknamed HyPERDBG, constructed entirely using the programming interface exposed by our infrastructure. HYPERDBG adds live and interactive debugging support to Microsoft Windows XP, so far only possible using very invasive tools, like Syser [?], or traditional VMM-based debuggers. HyPERDBG can be used to debug any component of the Windows kernel, including interrupt/exception handlers, device drivers, and even supports single instruction stepping. Being completely separated from the debuggee, HYPERDBG is transparent to the analyzed system and can be even used to analyze protected and malicious code.

In summary, the paper makes the following contributions.

1. We propose a framework to perform complex dynamic system-level analyses of commodity production systems. Compared to existing frameworks, the one we propose guarantees transparency, efficiency, and does not require the target system to be already installed on a virtual machine.
2. We implemented our framework in an experimental prototype for Microsoft Windows XP.

3. We describe the design and the implementation of $\mathrm{HY}$ PERDBG, a kernel-level interactive debugger built on top our framework.

Both the analysis framework and HYPERDBG are available at http://security.dico.unimi.it/hyperdbg/ and is released under the terms and conditions of the GPL (v3.0) license.

\section{RELATED WORK}

The framework proposed in this paper shares many similarities with frameworks and techniques extensively explored in the past. However, by exploiting recent facilities available of modern Intel x86 CPUs, our framework is able to combine and to offer simultaneously the main benefits introduced by previous research work.

\section{Dynamic Kernel Instrumentation.}

DTrace is a facility included into the Solaris kernel that allows the dynamic instrumentation of production systems [?]. The key points of DTrace are efficiency and flexibility. First, the instrumentation framework itself introduces no overhead. Second, the framework provides tens of thousands of instrumentation points, and the actions to be taken can be expressed in terms of a high-level control language, that also includes a number of mechanisms to guarantee runtime safety. Similarly, KernInst is a dynamic instrumentation framework for commodity kernels [?]. KernInst has been developed mainly to gather information about the performances of a running kernel, but it has also been employed for run-time kernel optimization. Differently from DTrace, KernInst does not provide any mechanism for runtime safety of the instrumentation routines. Unfortunately, the aforementioned approaches are not transparent, as they require direct modifications of the operating system kernel, achieved by loading a kernel-mode module. Moreover, none of them is OS-independent, and they and cannot be applied to closed-source operating systems. Our framework does not suffer these limitations since it can instrument the kernel without modifying it and does not rely on any facility offered by the kernel.

\section{Kernel-level Debugging.}

Several efforts have been made to develop efficient and reliable kernel-level debuggers. Indeed, these applications are essential for many activities, such as the development of device drivers. One of the first and most widely used kernel-level debuggers that targeted the Microsoft Windows operating system was SoftICE [?], but today the project has been discontinued. However, both commercial [?] and open-source [?] alternatives to SoftICE appeared. Modern versions of Windows already include a kernel debugging subsystem [?]. Unfortunately, to exploit the full capabilities of Microsoft's debugging infrastructure, the host being debugged must be physically linked (e.g., by means of a serial cable) with another machine. All these approaches share a common factor: to debug kernel-level code, they leverage another kernel-level module. Obviously, that is like a dog chasing its tail. The framework proposed in this paper does not require any kernel support nor to modify the kernel to add the missing support at run-time. 


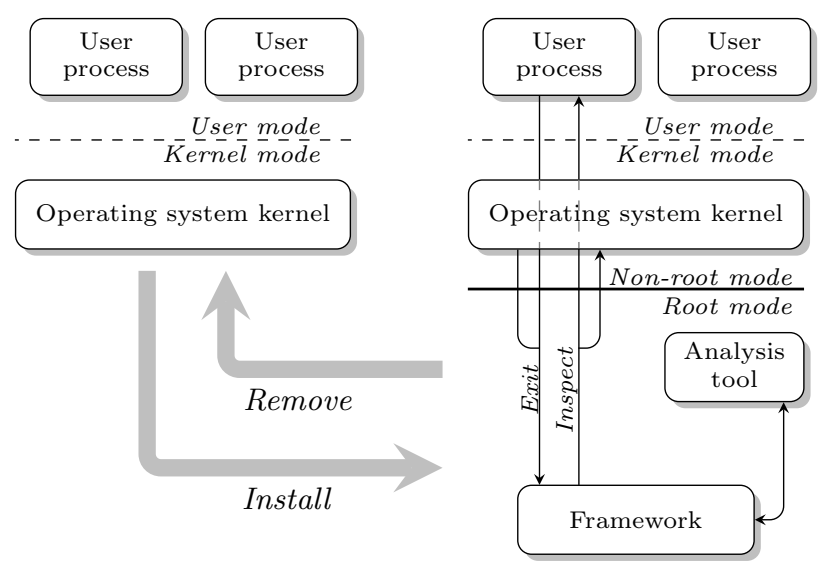

Figure 1: Overview of the framework

\section{Frameworks Based on Virtual Machines.}

Instead of relying on a kernel-level module to monitor other kernel code, an alternative approach consists of running the target code inside a virtual machine, and to perform the required analyses from the outside [?]. In [?, ?, ?] the authors propose virtual machines with execution replaying capabilities: a user can move forward and backwards through the execution history of the whole system, both for debugging and for understanding how a hacker intrusion took place. Finally, in [?] Chow et al. propose Aftersight, a system that decouples execution recording from execution trace analysis, thus reducing the overhead suffered by the system where the guest operating system is run. Nowadays, Aftersight is part of the VMware platform, and other mainstream commercial products provide similar capabilities. The framework proposed in this paper can provide these functionalities even on systems not running in any virtual machine.

\section{Aspect-oriented Programming.}

Aspect-oriented programming is a paradigm that promises to increase modularity by encapsulating cross-cutting concerns into separated code units, called "aspects", whose "advice" code is woven into the system automatically, by specifying the properties of the join-points. AspectC is an aspectoriented framework that is used to customize (at compiletime) operating system kernels [?, ?, ?]. More dynamic approaches have been proposed: for example TOSKANA provides before, after and around advices for in-kernel functions and supports the implementation of aspects themselves as dynamically exchangeable kernel modules [?]. The framework proposed in this paper allows to achieve the same goal while being transparent and fault-tolerant.

\section{OVERVIEW OF THE FRAMEWORK}

Figure 1 depicts the architecture of our framework, the installation and removal processes, and the migration of the operating system and its applications into a virtual machine. Our framework consists of a virtual machine monitor (VMM for short) that provides a programming interface for the development of system-level analysis tools. As in traditional VMM-based analysis approaches, the analysis tool is run within the VMM and thus completely transparent to guests of the virtual machine. However, compared to traditional
VMM-based ones, ours does not require the system to be already running inside any virtual machine. To achieve this goal, our framework leverages hardware extensions for virtualization available on all modern x86 CPUs [?, ?] (which are unused in the majority of the deployments). In short, these extensions augment the instruction set architecture with two new modes of operation: VMX root mode and VMX nonroot mode ${ }^{1}$. These new modes of operation separate logically the virtual machine monitor from a guest without having to modify the latter. More precisely, we exploit a particular feature of these extensions that allows for late launching of VMX modes. Late launching of VMX modes permits to install a virtual machine monitor even if the system has already been bootstrapped. In other words, late launching allows to migrate (temporarily) a running operating system in a virtual machine, and to analyze and control the execution of the system from the monitor. Through the rest of the paper, we use the term "guest" to refer to the system under analysis that has been migrated into a virtual machine.

Practically speaking, the running operating system is not migrated anywhere and not touched at all. Rather, by launching VMX modes, the execution environment is extended with the two aforementioned operating modes; the running operating system is then associated with non-root mode, while the VMM is associated with root mode. Thus, in all respects, the operating system and its applications become a guest of our special virtual machine. Following the same principle, the VMM can be unloaded, and the original mode of execution of the operating system restored, by simply disabling VMX modes. After the launch of the VMX modes, the execution of the guest can continue exactly as before, even in terms of interactions with the underlying hardware devices. However, during its execution, the guest might be interrupted by an exit to root mode. Like hardware exceptions, exits are events that block the execution of the guest, switch from non-root mode to root mode, and transfer the control to the VMM. Differently from exceptions, the set of events triggering exits to root mode can be configured dynamically by the VMM. A routine of the VMM handles the exit and eventually enters non-root mode to resume the execution of the guest. Being executed at the highest privilege level, the routine handling the exit has complete read/write control of the state of the guest system (of both memory and CPU registers).

The framework itself does not perform any analysis. It is only responsible for handling a small set of exits to control all accesses to the memory management unit of the CPU, to prevent the guest from accessing the physical memory locations holding the code and the data of the framework. On the other hand, the framework provides a flexible API to develop tools to perform sophisticated analyses of both kernel and user code running in the guest. Using the functionalities provided through the API, the tool can request the framework to monitor certain events that might occur during the execution of the guest; when such events occur, it can inspect, and even manipulate, the state of the guest. The events that can be monitored include, but are not limited to, system call invocations, function calls, context switches and I/O operations. Practically speaking, events are monitored through exits to root mode. Thus, a request of the analysis tool to monitor a certain high-level event (e.g., the execu-

\footnotetext{
${ }^{1}$ VMX (non-) root mode is the terminology used by Intel; AMD adopts a different terminology.
} 


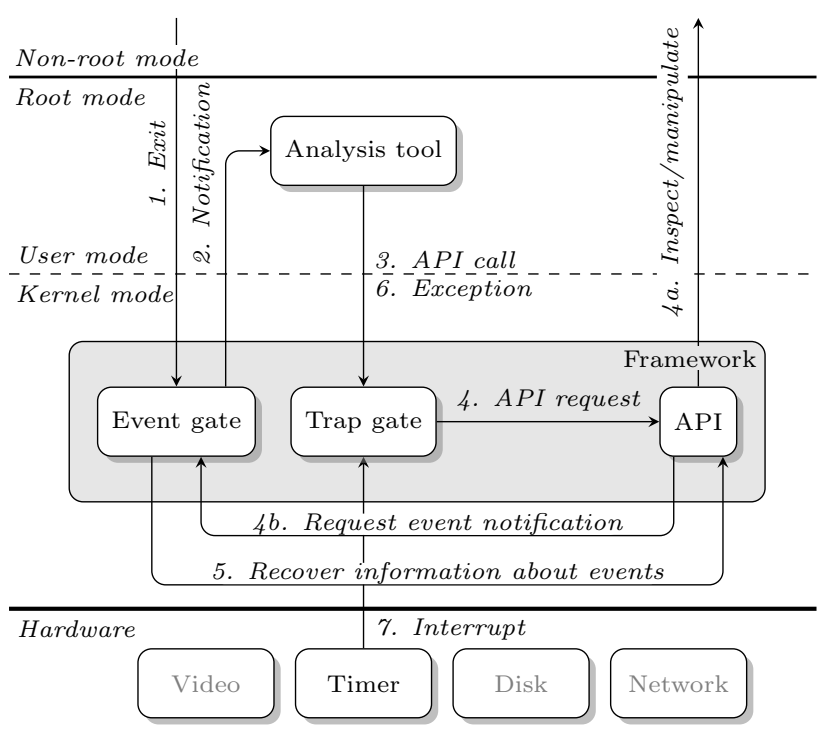

Figure 2: A close-up of the framework

tion of a system call) is translated by the API of the framework into a sequence of low-level operations that guarantee that all the occurrences of such event in the guest trigger an exit to root mode. Similarly, the framework translates the exit into a higher-level event and notifies the occurrence of the event to the analysis tool. Once notified, the tool can recover information about the event (e.g., arguments and return value of a system call), using the inspection functionalities offered by the API.

An important requirement for the analysis of production systems is that analysis tools must not interfere with the correct execution of the guest. This is particularly important for faults and deadlocks that might occur in the analysis tool. The approach we adopt is to run the tool in a less privileged execution environment, isolated from the analyzed system and from the framework. The tool can interact with the guest only through the API exposed by the framework. This approach guarantees the framework the ability to intercept any fault occurring in the tool, to mediate all accesses to the analyzed system (and to prevent write accesses), and to terminate the tool in case of deadlocks or other anomalous situations.

\section{DESIGN AND IMPLEMENTATION}

Figure 2 shows a more detailed view of the architecture of our framework. Intuitively, this architecture is very similar to that of traditional operating systems: the framework plays the role of the kernel and the analysis tool plays the role of a user-space application. As will become clear later, this architecture prevents buggy analysis tools from compromising the guest system and the framework. The separation between these two parts is made possible by the fact that, when VMX is enabled, root and non-root modes offer two fully-featured execution environments. Thus, like the guest running in non-root mode, the framework running in root mode can rely on privilege separation to isolate the analysis tool and can handle independently interrupts and exceptions that might occur while executing in root mode.

When an exit to root mode interrupts the execution of the guest, the event is delivered to the event gate (step 1 in Figure 2). The event gate is responsible for abstracting low-level events into higher-level ones, and to notify the analysis tool if the latter has requested to do so (step 2). On startup the analysis tool requests the framework to be notified of certain events (not shown in the figure). The tool can use the API provided by framework to query extra information about the event (e.g., the content of the stack location storing one of the arguments of a function). Since the tool is isolated from the framework, API functions are invoked through software interrupts. Thus, requests coming from the analysis tool are received by the trap gate (step 3 ), then forwarded to the component implementing the API (step 4). The tool can perform two types of API calls: (step 4a) to inspect or manipulate the state of the guest, and (step 4b) to control event notifications (e.g., enable or disable the notification of certain events). Note that the component implementing the API is also used by the framework itself (step 5) to recover extra information about events (e.g., the return address of a function stored in the stack). The trap gate also serves the purpose of detecting exceptions (e.g., page faults) that might occur during the execution of the analysis tool. If the trap gate intercepts an exception (step 6 ), it terminates the faulty tool and unloads the framework, to resume the normal operation mode of the system. Finally, the trap gate is also used to handle timer interrupts (step 7), that, as will be discussed in Section 4.4, are employed to enforce a time-bound on the execution of the tool.

The functionalities provided by the API of the framework can be classified into two classes: execution and I/O tracing and state inspection and manipulation. The following paragraphs describe briefly the API. More details are given in Sections 4.2 and 4.3 .

Execution and I/O tracing facilities allow a tool to intercept the occurrence in the analyzed system of certain events and certain I/O operations respectively. Table 1 reports the main types of events that can be traced. For each event, the table also reports the arguments associated to the event; arguments are information about the events most commonly used in tools. For example, the events FunctionEntry and SyscallEntry are used to trace functions and system calls respectively. The arguments associated to the FunctionEntry event are the address (or the name) of the function called, the caller and the return address. Another example is the ProcessSwitch event that can be used to trace context switches between processes (not threads). From the point of view of the analysis tool all the events are handled in the same way: the tool can subscribe to any event and, when the event occurs, can inspect its arguments and take the proper actions. However, at the framework-level, certain events are different from other ones. Indeed, some of them (e.g., context switches between processes) can be traced directly by the hardware. That is, the event triggering the exit corresponds exactly to the event being traced. Other events instead (e.g., function calls and returns) cannot be traced directly by the hardware. In all these cases the framework relies on other low-level events to trace the execution and then abstract exiting low-level events into higher-level ones, meaningful for the analysis tool.

Arguments can optionally be used as conditions, to limit the tracing to a subset of all the events. Conditions on events serve two purposes. First, conditions allow to simplify the analysis tools, since events that do not match the requested conditions are discarded by the framework and thus do not 


\begin{tabular}{lll}
\hline Event & Description & Arguments \\
\hline ProcessSwitch & Context (process) switch & - \\
Exception & Execution & Exception vector, faulty instruction, error code \\
Interrupt & Hardware or software interrupt & Interrupt vector, requesting instruction \\
BreakpointHit & Execution breakpoint & Breakpoint address \\
WatchpointHit & Watchpoint on data read/write & Watchpoint address, access type, hitting instruction \\
FunctionEntry & Function call & Function name/address, caller/return address \\
FunctionExit & Return from function & Function name/address, return address \\
SyscallEntry & System call invocation & System call number, caller/return address \\
SyscallExit & Return from system call & System call number, return address \\
IODperationPort & I/O operation throught hardware port & Port number, access type \\
IOOperationMmap & Memory-mapped I/O operation & Memory address, access type \\
\hline
\end{tabular}

Table 1: Events traceable using our framework and corresponding arguments (the argument that represents the current process is omitted, as it is common to all the events)

need to be handled by the tool. Second, some conditions allow preemptive filtering of the events. In other words, the framework configures a priori which events trigger an exit, instead of filtering out exits caused by uninteresting events. For example, in the case of the IOOperationPort event, preemptive filtering means to configure the CPU such that only I/O operations involving a specific I/O port trigger an exit. This feature is very important to minimize the number of exits and thus the overall overhead.

State inspection and manipulation primitives can be used by the tool to access the state of the guest, in order to extract more detailed information about events or other data useful for the analysis. For example, these primitives allow to extract the arguments of an invoked function, or to inspect the internal structures of the guest operating system. Note that, by default, write access to guest state is not granted to a tool. If necessary, such permission can be enabled at compile-time. Obviously, in this case the framework cannot protect the state of the guest from dangerous modifications.

\subsection{Framework and Analysis Tool Loading}

The framework and the analysis tool are loaded by a minimal kernel driver. This is unavoidable since the operations we need to perform to load the framework require maximum privileges and can be performed only by the kernel of the operating system. The driver, however, is indeed very simple and we put extreme care in avoiding any interference with the kernel. Moreover, since once loaded the framework is completely invisible to the system, we unload the driver immediately as soon as the framework has been installed.

When VMX modes are enabled, a special VMX data structure (VMCS in Intel terminology) is made accessible initially to the loader, and subsequently, when the loading is completed, only to the framework. This data structure stores the host state, guest state, and the execution control fields. The host state stores the state of the processor that is loaded on exits to root mode, and consists of the state of all the registers of the CPU (except for general purpose registers). Similarly, the guest state stores the state of the processor that is loaded on entries to non-root mode. The guest state is updated automatically at every exit, such that the subsequent entry to non-root mode will resume the execution from the same point. The execution control fields allow a fine-grained specification of which events should trigger an exit to root mode.

The task of the loader is to enable VMX modes and to configure the VMX data structure such that the execution of the operating system and user-space applications continue to run in non-root mode, while the framework and the analysis tool are executed in root mode. Moreover, the loader has to configure the CPU such that all the events necessary for the tool to trace the execution of the system trigger exits to root mode. When the initialization is completed, the driver unloads itself and resumes the execution of the system.

\section{Guest State Configuration.}

The guest state is initialized to the current state of the system. In this way, when the virtual machine is launched and execution enters non-root mode, the guest operating system will resume its execution as if nothing happened. A tricky problem when initializing non-root mode concerns the management of the memory. More precisely, we must prevent the newly created guest to use and access the physical memory frames allocated to the framework and to the tool. Otherwise, the guest could detect and even corrupt the framework. Most recent CPUs provide hardware facilities for memory virtualization (e.g., Intel Extended Page Table extension). If these facilities are not available, memory virtualization must be implemented entirely via software. Briefly, software memory virtualization consists of intercepting all guest operations to manipulate the page table (the data structure the CPU uses for virtual-to-physical address translation) and in ensuring that none of the physical frames allocated to the framework and to the analysis tool are mapped into the guest. In case the guest tries to map a reserved physical frame, the framework assigns the guest a different one and masquerades the difference.

\section{Host State Configuration.}

The host state is initialized as follows. The CPU is configured to use, when in root mode, a dedicated address space and a dedicated interrupt descriptor table (IDT). This configuration simplifies the separation of the analyzed system from the framework and allows to detect and handle interrupts and exceptions that occur in root mode. Differently from the address of the entry point of non-root mode, which is updated at every exit to allow to resume execution of the guest from where it was interrupted, the address of the entry point of root mode is fixed. The entry point is set to the address of the routine that takes care of dispatching an exit event to the appropriate handler and that in turn might notify the analysis tool (i.e., the entry point of the event gate).

\section{Execution Control Fields Configuration.}

To reduce the run-time overhead suffered by the guest 


\begin{tabular}{|c|c|c|}
\hline Event & Exit cause & $\begin{array}{c}\text { Native } \\
\text { exit }\end{array}$ \\
\hline ProcessSwitch & Change of page table address & $\sqrt{ }$ \\
\hline Exception & Exception & $\sqrt{ }$ \\
\hline Interrupt & Interrupt & $\sqrt{ }$ \\
\hline BreakpointHit & Debug except. / Page fault except. & \\
\hline WatchpointHit & Page fault except. & \\
\hline FunctionEntry & Breakpoint on function entry point & \\
\hline FunctionExit & Breakpoint on return address & \\
\hline SyscallEntry & Breakpoint on syscall entry point & \\
\hline SyscallExit & Breakpoint on return address & \\
\hline I0OperationPort & Port read/write & $\sqrt{ }$ \\
\hline IOOperationMmap & Watchpoint on device memory & \\
\hline
\end{tabular}

Table 2: Techniques for tracing events

system, the execution control fields are configured to minimize the number of events that trigger an exit to root mode. When the tool is initialized, it specifies which events must be intercepted. Subsequently, in response to the invocation of API functions, the configuration of the execution control fields can be altered to intercept additional events or to ignore other ones.

\subsection{Execution Tracing}

Table 2 describes the technique used to trace all the events currently supported by the framework. Low-level events (those with a mark in the last column) correspond directly to exits to root mode (e.g., Exception). Other events are traced through the aforementioned ones (e.g., BreakpointHit), and others again are traced through the latter (e.g., FunctionEntry).

Events that can be traced directly through the hardware are process switches, exceptions, interrupts, and port-based I/O operations. All these events exit conditionally: they exit to root mode only when requested and can have optional exit conditions to limit exits to particular situations. The remaining of this section presents how we developed the primitives for tracing higher-level events starting from the aforementioned low-level ones.

Breakpoints and watchpoints are two of the most complicated events to implement. Modern CPUs provide hardware facilities to realize efficient and transparent breakpoints and watchpoints. Unfortunately, hardware-assisted breakpoints and watchpoints are limited in number (only 4) and shared between non-root and root mode. Therefore, they cannot be used simultaneously by the analyzed system and by the framework. The solution we adopt to allow an arbitrary number of breakpoints is to use software breakpoints. A software breakpoint is a one-byte instruction that triggers a breakpoint exception when executed. Software breakpoints are enabled by replacing the byte at the address on which we want the breakpoint with the aforementioned instruction. When the breakpoint is hit, the original byte is restored and the event is notified to the tool. If the breakpoint is not persistent the execution of the system is resumed. Otherwise the instruction is emulated and then the breakpoint is set again. Clearly, this approach to breakpoints is not transparent for the analyzed system. However, it is very efficient. An alternative and transparent approach is to use the same technique we use for watchpoints, as described in the next paragraph. Our framework supports both approaches.

The approach used in our framework to implement software watchpoints is based on protecting the memory locations from any access via hardware (or just from write accesses, depending on the type of watchpoint), such that any access results in an exception [?]. More precisely, since the finest level of protection offered by the hardware is at the page level, we mark the page containing the address on which we want to set the watchpoint as "non-present". Any future access to this page will result in a page fault exception that will be intercepted by our framework. The framework analyzes the exception and checks whether the accessed address corresponds to the address with the watchpoint. If the watchpoint is hit, the framework delivers the event to the analysis tool, otherwise it emulates the instruction, and then resumes the normal execution of the guest. Emulation is necessary to execute the faulty instruction manually. Indeed, to prevent a second fault, the original permission of the memory page accessed by the instruction must be restored before executing the faulty instruction. After the execution of the instruction, the page must be marked again as "non-present" to catch future accesses.

Other higher-level events, such as function and system call entries and exits, are traced through breakpoints. When the analysis tool requests the framework to monitor a certain function, the framework sets a breakpoint on the address of the entry point of the function. Later, when a breakpoint is hit, the framework checks whether the hit breakpoint corresponds to a function entry point and, if so, it delivers the appropriate event (i.e., FunctionEntry) to the analysis tool. Function exits, instead, are traced by setting a breakpoint on the return address. The framework discovers the return address by setting a breakpoint on the function entry and by inspecting the stack frame of the function when the breakpoint on the entry point is hit. A similar approach is used for tracing system calls entries and exits.

The approach for tracing function calls and returns just described allows to trace specific functions, whose names or addresses are supplied by the tool. The tracing of all function calls and returns is instead more complicated because it is not possible to know a priori the addresses of all functions' entry points. The solution in this case is to perform a static analysis to identify the addresses of all functions' entry points (e.g., by recognizing function prologues). This feature is still not available in our current implementation of the framework. Nevertheless, if needed, the static analysis could be performed directly in the tool. The tracing of all system calls is instead much easier, since they are all invoked through a common gate. The solution we adopt is to put a breakpoint on the entry point of the system call gate [?].

Beside execution tracing facilities, the framework also exposes to analysis tools the possibility of intercepting I/O operations with hardware peripherals. Software can interact with hardware devices through hardware I/O ports, or it can leverage memory-mapped I/O. In the first case, VMX allows to intercept the operation without any effort: the framework simply configures the execution control fields such that all the interactions with the specific hardware ports trigger an exit to root mode; when such an exit occurs, the framework notifies the tool by means of a I00perationPort event. However, for performance reasons, modern peripherals typically resort to memory-mapped I/O. In this case, read and write operations do not involve any hardware port, as they are performed directly on memory. To intercept such operations we set a watchpoint on the appropriate memory region. Thus, when an access to it is detected, the framework delivers a I00perationMmap event to the tool. 


\subsection{State Inspection and Manipulation}

Several situations require to access the state of the guest system in order to inspect, and optionally manipulate, both the registers of the CPU and the memory. As an example, the framework could need to read the return address of a function from the stack, to access the parameters of a system call from the processor registers, or to insert a breakpoint into the address space of a particular process. Similarly, the analysis tool might need to extract data from the memory of the guest.

The inspection and manipulation of $\mathrm{CPU}$ registers is a straightforward activity. These information are saved during an exit and restored before an entry. Thus, the inspection and manipulation of registers merely consists of reading or writing the VMX guest state (or the memory of the framework, depending on the type of register).

Inspection and manipulation of memory locations is much more complex. When paging is enabled, virtual addresses are translated by the hardware into physical addresses according to the content of the page table and direct physical addressing is not possible. Each process has its own page table; therefore, different processes have different virtual-tophysical mappings and a process cannot access the memory of the others. The framework is isolated from the guest using the same approach and thus it has its own page table and its own mapping. Consequently, the framework cannot directly access memory locations of guest processes. Moreover, inspection is complicated by the fact that page tables cannot be traversed via software (but only via hardware): the page table is a multilevel table and pointers to lower levels are physical. To overcome this problem we have developed a specific, OS-independent, algorithm that allows to access an arbitrary virtual memory location of an arbitrary process. The core of the algorithm is a primitive that allows to access arbitrary physical memory locations. This is accomplished by mapping a given physical address $p$ to an unused virtual address $v$ in the page table of the framework, and subsequently by accessing $v$. Then, using this primitive, the algorithm can traverse the page table of a process of the guest via software by iteratively mapping the physical addresses stored in the table.

The framework exposes memory inspection and manipulation facilities, based on the aforementioned algorithm, to the analysis tools through two API functions: GuestRead $(p, a,-$ $n$ ) and GuestWrite $(p, a, \operatorname{dat} a)$. The former reads $n$ bytes starting from virtual address $a$ of process $p$; the latter writes the content of buffer data into the address space of process $p$, starting from virtual address $a$. By default, to preserve the integrity of the guest, all GuestWrite operations are forbidden. On top of this functions we have built higher-level ones that facilitates the extraction of functions' arguments, null terminated strings, and to disassemble code.

\subsection{Tool Isolation}

To be able to use our infrastructure on a production system, it is essential to guarantee that any defect in the analysis tool will not affect the stability of the analyzed system and of the framework. At this aim, the framework controls the execution of the analysis tool and, if any anomalous behavior is observed, the whole infrastructure is automatically unloaded.

As we outlined at the beginning of this section, even if the analysis tool is executed in VMX root mode, it is still constrained into a less privileged execution mode than the framework. Thus, any operation the tool performs on the guest must be mediated by the framework. This is exactly what happens in traditional operating systems: a user-mode process cannot access directly the resources of the operating system, nor those of other user-mode processes, and any action it performs outside its address space must be mediated by the kernel. Similarly in our context, to perform an operation on the guest system, the tool must use the programming interface offered by the framework.

In the default configuration, the framework does not allow a tool to access in write-mode to the state of the guest. However, there is still the possibility that the execution of an instruction of the tool raises an unexpected exception (e.g., a page fault on memory access, or a general protection fault). When such an event occurs, the framework has no way to handle the anomalous situation and to allow the tool to continue its execution. The only viable approach that also preserves the integrity of the guest system is to terminate the analysis tool and to remove the framework. At this aim, the solution we adopt is to intercept unexpected exceptions through the custom interrupt descriptor table (IDT) installed when launching VMX modes. The IDT receives the trap, and delivers it to the trap gate that eventually unloads the framework. Another problem that might arise with a buggy analysis tool is non-termination: if the analysis tool entered an infinite loop, the guest system would never be resumed. To prevent this problem we added to the framework a minimalistic watchdog and set a time limit on the execution of the tool. The limit is not on the whole execution time of the tool, but rather on the execution time to handle an event. Thus, the analysis tool could potentially be run forever, but with the guarantee that the execution of the analyzed system will be resumed within the specified time limit. At this aim, before delivering an event to the analysis tool, the framework resets a timer. Then, while the tool handles the event, the framework periodically regains the control of the execution and checks whether the time limit has been exceeded. To do that the framework registers, in the IDT, a custom interrupt handler to handle timer interrupts and programs the interrupt controller to deliver only timer interrupts (that is necessary to prevent the framework to consume interrupts for all the other devices). Before returning to non-root mode, the framework reprograms the interrupt controller to deliver all the interrupts to the analyzed system.

\subsection{OS-dependent Interface}

Our framework provides a general programming interface completely independent from the operating system running inside the guest. However, in many cases some OS-specific facilities can ease the analysis of the guest. As an example, the only OS-independent manner to identify a process is by means of the base address of its page table (typically stored inside the cr3 CPU register). However, it is quite awkward to refer to processes using page table base addresses, and it is more natural to identify a process through its process id (PID) or through the name of the application it executes.

The OS-dependent interface we provide leverages virtual machine introspection techniques [?] to analyze the internal structures of the guest operating system to translate OS-independent information (e.g., process with page table base address 0x13cdc000) into something more user-friendly 


\begin{tabular}{|c|c|}
\hline Name & Description \\
\hline GetFuncAddr $(n)$ & Return the address of the function $n$ \\
\hline GetFuncName $(a)$ & Return the name of the function at address $a$ \\
\hline $\operatorname{GetProcName}(p)$ & $\begin{array}{l}\text { Get the name of process with page directory } \\
\text { base address } p\end{array}$ \\
\hline $\operatorname{GetProcPID}(p)$ & $\begin{array}{l}\text { Get the PID of process with page directory } \\
\text { base address } p\end{array}$ \\
\hline $\operatorname{GetProcLibs}(p)$ & $\begin{array}{l}\text { Enumerate the dynamically linked libraries } \\
\text { loaded into process } p\end{array}$ \\
\hline $\operatorname{GetProcStack}(p)$ & Get the stack base for process $p$ \\
\hline $\operatorname{GetProcHeap}(p)$ & Get the heap base for process $p$ \\
\hline GetProcList() & Enumerate processes \\
\hline GetDriverList() & Enumerate device drivers \\
\hline
\end{tabular}

Table 3: OS-dependent API

(e.g., process notepad.exe). Moreover, using debugging symbols, the framework allows to resolve symbols' names and addresses (e.g., functions and global variables). In this way, a tool can ask to interrupt the execution of the guest when function NtCreateFile is invoked, instead of referencing the function through its address. Similarly, when a function is invoked, it is possible to inspect its call-stack and to resolve the name of the caller functions and even recover the libraries to which the various functions belong to. Some of the OS-dependent functionalities provided are summarized in Table 3.

In case the guest operating system is not supported, the OS-dependent module is disabled, and only OS-independent functionalities are available. Our current implementation offers an OS-dependent interface only for the Windows XP operating system.

\section{APPLICATIONS}

In this section we present HYPERDBG, an interactive kernel debugger for Microsoft Windows XP we built on top of our framework. In our strive to contribute to the open source community, we released the code of HYPERDBG, along with the code of the framework, under the GPL (v3.0) license. The code is available at the following address:

\section{http://security.dico.unimi.it/hyperdbg/}

The section also discusses other possible applications that could be constructed using our framework.

\subsection{HyperDbg}

HyPERDBG is an interactive kernel debugger we developed on top of our analysis framework. It offers all the features commonly found in kernel-level debuggers but, being completely run in VMX root mode, it is OS-independent and grants complete transparency to the guest operating system and its applications. The debugger provides a simple graphical user interface to ease the interaction with the user. This interface is activated in two circumstances: (I) when the user presses a special hot-key or (II) when the debugger receives the notification for an event that requires the attention of the user (e.g., when a breakpoint is hit). From this interface the user interacts with the debugger and can perform several operations, including setting breakpoints and watchpoints, tracing functions and system calls, and inspecting and manipulating the state of the guest (since all interactive debuggers allow to modify the state of the debuggee, we decided to enable write access to the guest as well).

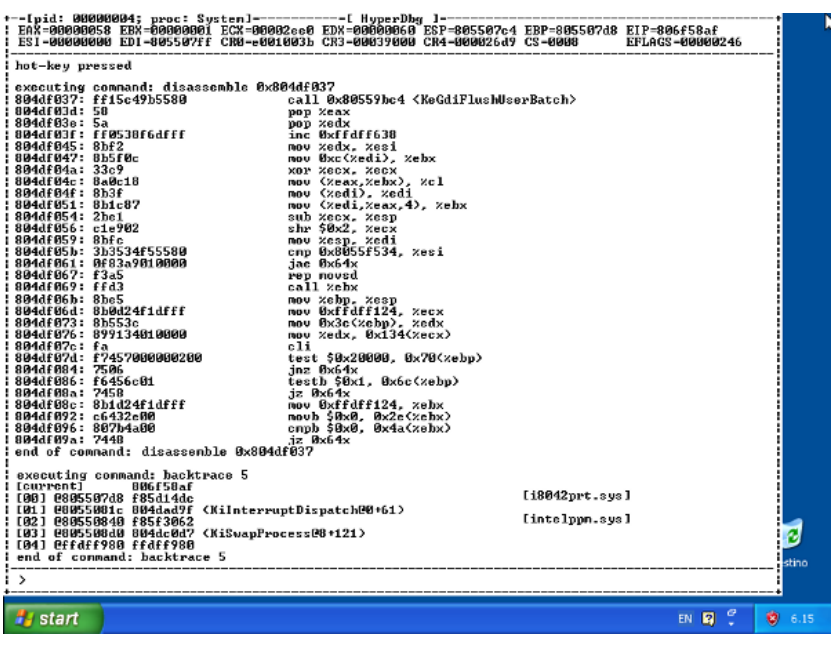

Figure 3: HyperDbg in action

Figure 3 shows HyPERDBG in action ${ }^{2}$. In particular, the figure shows the debugger notifying the event that interrupted the execution of the analyzed system, displaying a fragment of the code of the process currently running in the analyzed system and displaying a "backtrace" of the function calls that are currently active. Additionally, the debugger displays information about the status of the registers at the time the event occurred (in the case of the figure the event is the pressure of the hot-key). To facilitate the analysis, the debugger leverages OS-dependent information. For example, the screenshot in Figure 3 shows that the debugger resolved the ID and the name of the process in a MS Windows $\mathrm{XP}$ guest, by knowing how the process table is managed by the operating system.

It is worth pointing out that HYPERDBG can be used to debug any piece of code of the guest system, including critical components such as the process scheduler, or interrupt and exception handlers. Indeed, Figure 3 shows that the guest operating system has been stopped while executing the PS/2 keyboard/mouse driver (i8042prt.sys). Thanks to the fact that the framework on which the debugger is built on is completely transparent to the analyzed system, the user can use the keyboard to interact with the debugger even though the keyboard driver of the guest is being debugged.

HYPERDBG consists of less than 1600 lines of code: $\sim 25 \%$ of the code implements the graphical interface, $\sim 23 \%$ of the code provides the facilities required for keyboard-based user interaction, and the remaining $\sim 52 \%$ is responsible for handling events and for all the other interactions with the framework. Note that certain functionalities (e.g., disassembling a code region) are implemented directly in the framework since, most likely, they will be used for other types of analysis as well. The framework is about four times bigger than the debugger (without considering the disassembly module embedded in the framework, as it is based on an off-the-shelf disassembler). We believe these numbers are very significant. The number of lines of code we had to write to implement HYPERDBG clearly witnesses that com-

\footnotetext{
${ }^{2}$ The screenshot was taken using our development environment based on an Intel x86 emulator supporting extensions for virtualization (i.e., BOCHS).
} 
plex analysis tools like an interactive kernel debugger are straightforward to implement using our framework.

The remaining of this section describes how we used the facilities of the framework to implement the user interface and the component to receive commands from the user.

\section{User Interface.}

Although the graphical user interface of the debugger is rough, its implementation is very challenging. The reason of the complexity is the fact that we cannot rely on any high-level graphical facility available in the analyzed system to render the interface. Such approach would be too OSdepended and not transparent at all. The lack of graphical primitives obliged us to interact directly with the video card. The video memory is mapped at a fixed address in the guest and thus unmodified inspection and manipulation API (i.e., GuestRead and GuestWrite) can be used by the debugger to render the interface. Note that this approach is not dependent on the OS nor on the hardware. We developed a small video library that provides basic graphical functionalities and translates our requests into data that are written directly in the memory of the video card. Before rendering the graphical interface to the screen, the debugger backups the content of the video memory and restores the content right before resuming the execution of the analyzed system.

\section{User Interaction.}

User interaction is keyboard-based. When in non-root mode, the user can switch into HYPERDBG by pressing a hot-key. Then, in root mode the user can control the debugger. For these reasons, HyPERDBG must be able to intercept keystrokes both in root and non-root mode. To intercept keystrokes in non-root mode we monitor all the read operations from the hardware I/O port devoted to the keyboard. In other words, HYPERDBG registers to the core for all the IOOperationPort events that satisfy the event condition port $=$ KEYBOARD_PORT \&\& access=read. When such operation is detected, HYPERDBG checks whether the key pressed corresponds to the hot-key that enables the debugger. If the key pressed matches the hot-key the debugger pops up the graphical interface and waits for commands. Otherwise, the debugger passes the keystroke to the analyzed system such that the latter will continue its execution as if the keystroke were read directly from the keyboard. Keyboard handling in root mode is done by polling the keyboard hardware I/O port. Since direct access to I/O ports is not permitted to any analysis tool, the debugger relies on a API function exported by the framework which mediates all accesses to I/O ports and allows (if the permission is granted at compile time) certain analysis tools to read data from certain I/O ports.

\subsection{Other Possible Uses of the Framework}

HYPERDBG demonstrates that our framework is very versatile and that enables new opportunities for dynamic analysis and we will explore in our future research.

An interesting extension of HYPERDBG will be the support for kernel-level omniscent debugging. Omniscent debugging allows developers to inspect the status of their programs in past execution instants, in order to detect the cause of a failure without the need to run the target program multiple times [?]. HyPERDBG could be extended to allow a user to record and inspect the values a memory location stored during the time, and the exceptions and interrupts occurred. Such a feature would ease a user to discover when a memory location of the kernel gets corrupted and which instruction is responsible for the corruption. Moreover, the ability to log asynchronous events, such as interrupts, would allow to spot defects connected to non-deterministic behaviors of the analyzed system. Our framework already offers all the necessary facilities for this kind of debugging: exception and interrupts can be traced natively by the framework and memory accesses can be traced using watchpoints.

Another interesting application of our framework will be dynamic aspect-oriented programming of operating system kernels. As discussed in Section 2, several approaches have been proposed to apply AOP to kernels. The main advantage offered by our framework over the approaches proposed so far is that it does not require any modification of the source code of the kernel, nor any modification of the image in memory of the kernel. Moreover, our framework protects the running kernel from defects in the woven code. One approach to facilitate the use of such technology would be to provide programmers a source-to-source translator, to translate aspect oriented code written in languages like AspectC [?] into $\mathrm{C}$ code that uses the API offered by our framework. In particular, the translator would be responsible for translating pointcuts into API calls to trace the corresponding events, using advices as events handlers, and for translating all pointer dereferences into calls to inspection API to read the memory of the guest.

\section{CONCLUSIONS}

We proposed a framework to perform complex run-time analyses of both system- and user-level code on commodity production systems. The framework exposes an API that eases the development of analysis tools on its top. The approach we described leverages hardware extensions for virtualization available on modern processors to overcome the limitations that affect existing approaches for the analysis of system-level code. In particular, the solution we proposed does not require to recompile or reboot the target system, it is not invasive, it is almost completely OS-independent, and it guarantees that a defect in an analysis tool cannot damage the framework itself nor the analyzed system. To demonstrate its potentials, we developed HyPERDBG, an interactive kernel-level debugger for Microsoft Windows XP. HYPERDBG and the framework have been released as an open source package.

\section{Acknowledgments}

This research has been partially funded by the European Commission, Program IDEAS-ERC, Project 227977 SMSCom and by the Italian Ministry of Education, Universities and Research, Program PRIN-2008. 